\title{
Defects in p-GaN and their atomic structure
}

\author{
Z. Liliental-Weber, T. Tomaszewicz, D. Zakharov, J. Jasinski and M. O’Keefe \\ Lawrence Berkeley National Lab, Berkeley, CA
}

\begin{abstract}
In this paper defects formed in p-doped GaN:Mg grown with Ga polarity will be discussed. The atomic structure of these characteristic defects (Mg-rich hexagonal pyramids and truncated pyramids) in bulk and thin GaN:Mg films grown with Ga polarity was determined at atomic resolution by direct reconstruction of the scattered electron wave in a transmission electron microscope. Small cavities were present inside the defects. The inside walls of the cavities were covered by GaN which grew with reverse polarity compared to the matrix. It was proposed that lateral overgrowth of the cavities restores matrix polarity on the defect base. Exchange of $G a$ and $N$ sublattices within the defect compared to the matrix lead to a $0.6 \pm 0.2 A$ displacement between the Ga sublattices of these two areas. A [1100]/3 shift with change from AB stacking in the matrix to $B C$ within the entire pyramid is observed
\end{abstract}

\section{Introduction}

P-doping of GaN remains still difficult and not well understood. $\mathrm{Mg}$ remains the most efficient $\mathrm{p}$ dopant but the free hole concentration is limited to $2 \times 10^{18} \mathrm{~cm}^{-3}$ for $\mathrm{Mg}$ concentrations in the low $10^{19} \mathrm{~cm}^{-3}$ range. This could limit further development of $\mathrm{GaN}$ based devices. Further increase of the $\mathrm{Mg}$ concentration, up to $1 \times 10^{20} \mathrm{~cm}^{-3}$ leads to a decrease of the free hole concentration. This is commonly interpreted as auto-compensation due to increased formation of $\mathrm{N}$ vacancies or vacancy complexes with $\mathrm{Mg}$ [1]. Another characteristic feature is the occurrence of the so-called "blue band" in the photoluminescence spectra of highly Mg-doped GaN grown by MOVPE [2].

\section{TEM studies of defects in bulk and MOCVD grown GaN:Mg layers}

Transmission electron microscopy (TEM) studies show the formation of different types of $\mathrm{Mg}-$ rich defects, both in bulk crystals grown from Ga excess under high N-pressure and in crystals grown by MOCVD [3-6]. The types of the defects strongly depend on crystal growth polarity (Fig. 1a). For bulk crystals grown with N-polarity, the planar defects (shown on Fig. 1a as lines) are distributed at equal distances (20 unit cells of GaN). These defects were described as inversion domains [6].

(a)

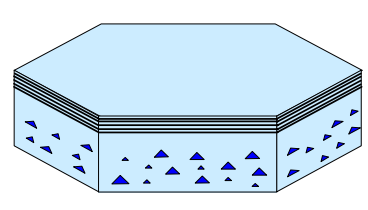

(b

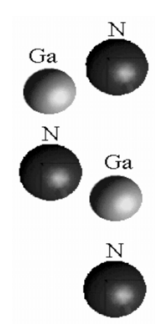

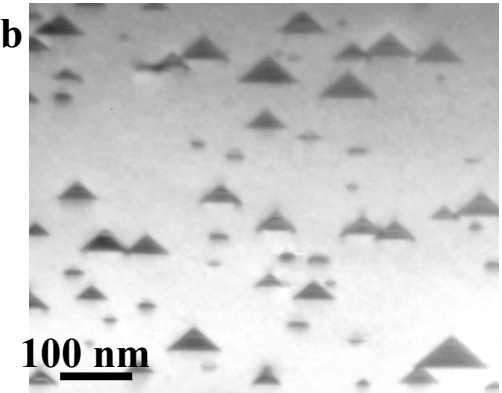

Fig. 1. (a) Schematic drawing shows defect distribution in a bulk GaN samples together with atom arrangement indicating crystal polarity; (b) TEM micrograph of defects in [1120] projection.

For growth with Ga-polarity (for both bulk and MOCVD grown crystals) two different types of defects have been found [4-6]. One, three-dimensional Mg-rich hexagonal pyramids with their base on the (0001) plane and six walls formed on $\{1123\}$ planes and the second, truncated pyramids (Fig. 1b). TEM micrographs of these defects in cross-section and plan-view are shown on Fig. 2(a-f). The direction from 
the tip of the pyramid to its base is along the Ga to $\mathrm{N}$ matrix bond direction [compare Fig. 2a and Fig. 2g]. For the trapezoidal features (Fig. 2b) a direction from the shorter to the longer base is also along the same $\mathrm{Ga}$ to $\mathrm{N}$ matrix bond direction. It appears that the dimension of these pyramids varies depending on growth method, but the angle between the base and their sides remain the same. In the bulk crystals the diameter of the base can be almost one order of magnitude larger (up to $1000 \AA$ ) than in thin foils grown

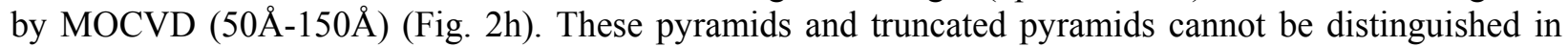
plan-view configuration indicating their common origin. Our studies show formation of small $\mathrm{Ga} / \mathrm{Mg}$ clusters near the tip of the pyramid [7]. It is possible that such clusters are origins of these defects. Depending on the cluster size the defect develops either in a pyramid or truncated pyramid.

(e)
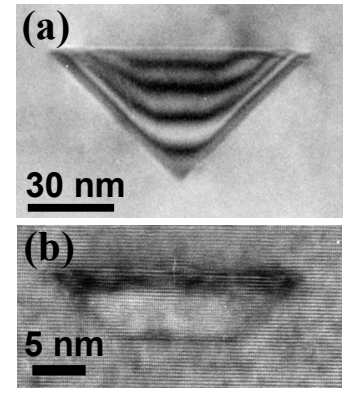

(c)

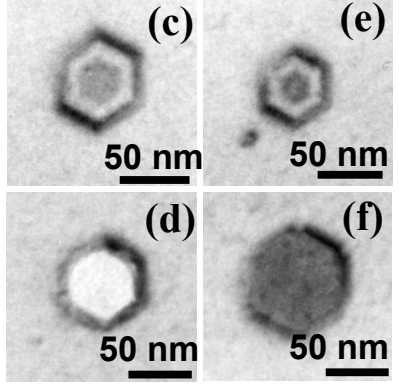

(e)

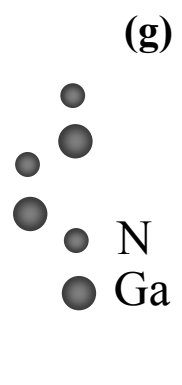

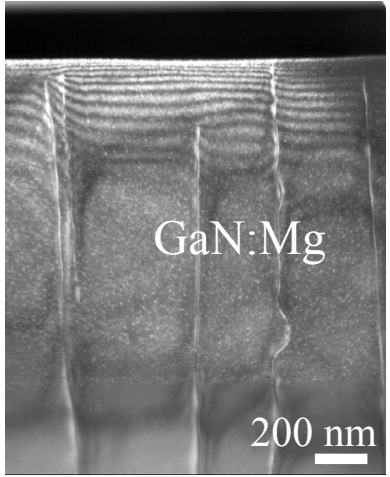

(h)

Fig. 2. TEM micrographs of two types of defects observed in cross-section bulk GaN:Mg samples: (a) a pyramid seen as triangular defect, (b) a truncated pyramid is seen as a trapezoid. (c-f) Same defects observed in plan-view samples. Their shapes indicate different sectioning in the plan-view TEM sample. (g) Atomic arrangement showing matrix Ga polarity [0001]. MOCVD GaN:Mg layer (h). Small dots indicate discussed defects.
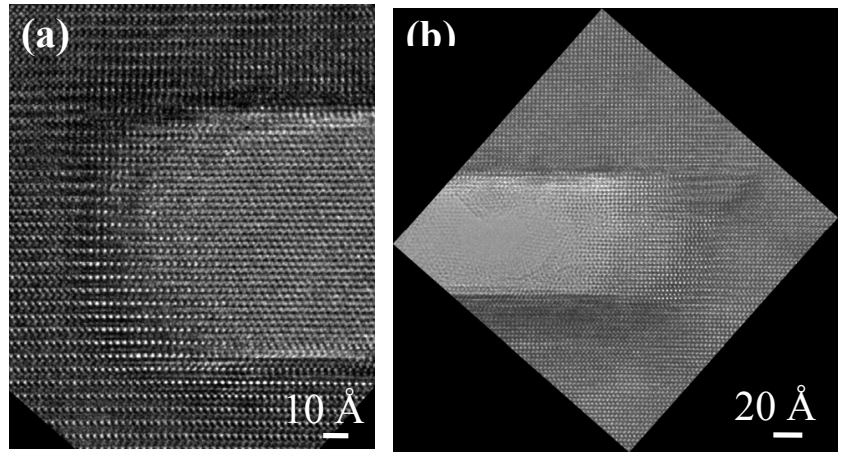

Fig. 3. High resolution image of two trapezoidal defects (only part of them are shown) showing different thickness within the defect and in the matrix (a) and a hole within the defect (b).

These both defects have often holes in their centers (Fig. 3) and are decorated by Mg on the defect walls [4-5]. Presence of such holes was also confirmed by positron annihilation study [7]. An expansion of the interplanar spacing along the c-axis was measured on the defect base, suggesting $\mathrm{Mg}$ accumulation [6]. Since these defects were also described as inversion domains [8] or $\mathrm{Mg}_{3} \mathrm{~N}_{2}$ precipitates [9] high resolution focal series for focal-series reconstruction of the electron exit-surface wave (ESW) leaving the specimen were applied for these studies [10]. The reconstructed exit surface wave is a complex function of position, with phase peaks that correspond to atoms positions. With appropriate measurements, one can distinguish between different elements contributing to the generated phase and define the number of atoms in the column. Since heavier atoms produce more phase change, in $\mathrm{GaN}$ the phase change produced by a Ga atom column is always stronger than the phase change produced by a $\mathrm{N}$ 
column with the equivalent number of atoms (Argant plot). Since the change of phase for Ga is much faster than for $\mathrm{N}$ for the same number of atoms in the column, we can distinguish between $\mathrm{Ga}$ and $\mathrm{N}$ columns, determine crystal thickness and the crystal polarity.

Reconstructed exit wave phase from the area close to the pyramid tip (Fig. 4) showed blobs with two distinct intensity peaks, identified as due to $\mathrm{Ga}$ and $\mathrm{N}$ atomic columns. Using Argand plots, we confirmed that the phase of atoms described as Ga gave the highest peak, followed by a smaller phase peak described as $\mathrm{N}$, as expected from earlier theoretical calculations. This identification of the atomic positions of $\mathrm{Ga}$ and $\mathrm{N}$ from the experimental reconstructed exit wave confirmed inversion of polarity within the pyramid compared to the matrix. In addition, it showed AB stacking in the matrix changes to BC stacking within the pyramid (Figs. 4b-d). This stacking arrangement holds through the entire pyramid and changes back to the $\mathrm{AB}$ stacking order above the pyramid base. Analysis of the reconstructed exit wave phase image from the pyramid side (along c planes) indicates a shift of $\mathrm{Ga}$ atomic column positions from the matrix to the $\mathrm{N}$ position within the pyramid. In this way a $0.6 \AA \pm 0.2 \AA$ displacement can be measured on the pyramid side between Ga positions in the matrix and within the pyramid.
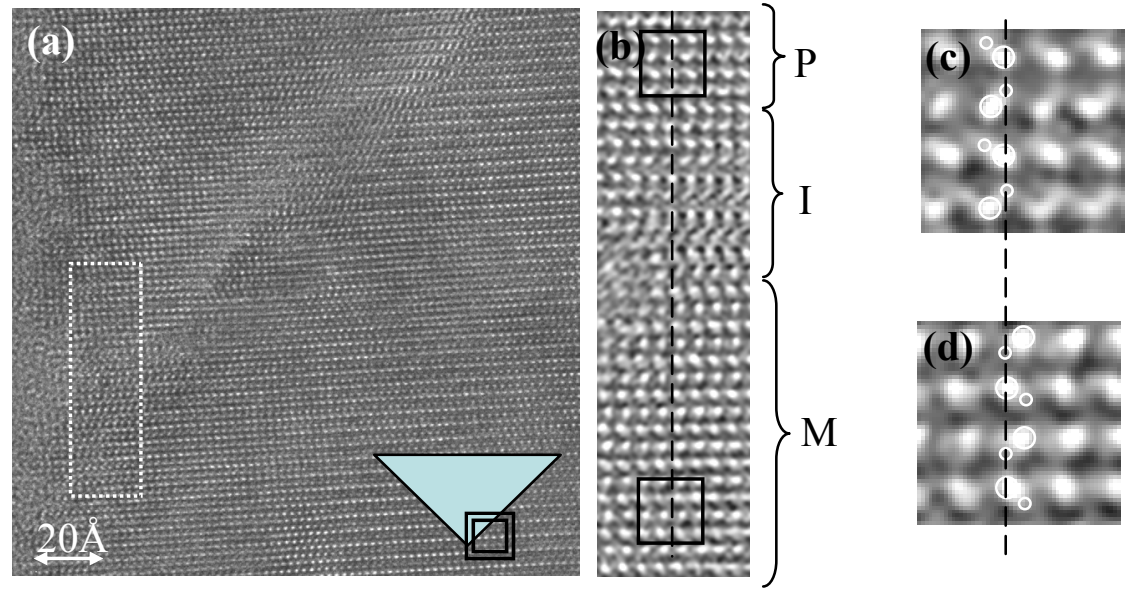

Fig. 4. Cross-section image from the part of pyramid close to the pyramid tip, schematically shown as inset in (a), (b) reconstructed exit wave phase from the side interface: P-inside the pyramids, I-the side interface area, M-the matrix below the side interface, (c) and (d) magnified reconstructed exit wave phase from the marked areas shown in (b) from the pyramid and the matrix, respectively, in high magnification with indicated atom position. Note stacking change within the defect (BC) compared to the matrix (AB).

Decoration by $\mathrm{Mg}$ was observed on the defect base and defect walls but not further from these interfaces excluding formation of $\mathrm{Mg}_{3} \mathrm{~N}_{2}$ precipitates suggested earlier [9]. Analysis of reconstructed exit wave phase on the defect base shows that $\mathrm{N}$ positions were occupied by different type of atoms. Since these atomic columns had phases smaller than the surrounding Ga columns above the base, they could be due to the presence of $\mathrm{Mg}$ in this part of defect. This is consistent with a larger separation between cplanes measured across the base. Increase in separation starts at $0.2 \AA$ above the pyramid and can grow, in some cases, up to $0.6 \AA$ at the pyramid base. Our Electron Energy Loss spectroscopy also indicates presence of oxygen [7] on the base and side walls and a change of shape of NK $\alpha$ edge which suggests different crystal structure. This observation and an expansion of the c-planes would be consistent with the decoration of several planes close to the base by $\mathrm{Mg}$ or $\mathrm{MgO}$. This compound could be responsible for the change of shape of NK $\alpha$ edge.

Our observations show very sharp base interfaces and well defined angles between the pyramid side and its base. We believe that the polarity change initiates at $\mathrm{Ga} / \mathrm{Mg}$ clusters, which initiate the growth of the defects. Considering the $43^{\circ}$ and $47^{\circ}$ angles between pyramid sides observed in 1120 and 1100 respectively, one can notice that the side plane is equally occupied by $\mathrm{Ga}$ and $\mathrm{N}$, therefore $\mathrm{Mg}$ can be 
attracted by both these atoms. Once the stacking sequence and polarity change at the pyramid side walls, this sequence will propagate to the base, changing again to the matrix arrangement above the base. Growth of GaN with the opposite polarity $(\mathrm{N})$ would be much slower leading to meniscus formation. A minute lack of $\mathrm{Mg}$ on the defect walls would lead to fast lateral overgrowth along c-planes, since this growth direction is much faster than along c-direction. Since $\mathrm{Mg}$ always floats on the surface, some rearrangement of $\mathrm{Mg}, \mathrm{Ga}$ and $\mathrm{N}$ would take place below the base (inside the pyramid), therefore it is not surprising to observe one or more c-planes rich in $\mathrm{Mg}$ close to the defect base. This lateral overgrowth would ensure an abrupt interface on the defect base and a return to the matrix polarity.

\section{Summary}

Transmission electron microscopy and reconstructed exit wave phase imaging were applied to study defects formed in bulk and MOCVD GaN:Mg crystals grown with Ga polarity. Two types of defects have been found: hexagonal pyramids with 6 inclined walls and a base formed on c-plane, and truncated pyramids. These defects have a hollow core, but their walls are covered by a substantial thickness of GaN grown with opposite polarity. These imaging results were confirmed by EELS and positron annihilation studies. Exchange of a Ga sublattice with a N sublattice within the defect leads to $0.6 \pm 0.2 \AA$ displacement between the Ga sublattices outside and inside the defects. Lateral overgrowth of the cavities formed within the defect is proposed to take place to ensure a return to matrix polarity on the defect base.

This work was supported by the U.S. Department of Energy under contract DE-AC0376SF00098. Use of the OAM facility at the National Center for Electron Microscopy at the LBNL is greatly appreciated.

\section{References:}

1. U. Kaufman, P. Schlotter, H. Obloch, K. Kohler, M. Maier, Phys. Rev. B62, 10867 (2002).

2. S. Nakamura and G. Fasol, "The blue laser diode" (Springer-Verlag, Berlin (1997).

3. Z.Liliental-Weber, M. Benamara, J. Washburn, et al, Phys. Rev. Letter. 82, 2370 (1999).

4. Z. Liliental-Weber, M. Benamara, W. Swider, J. Washburn, I. Grzegory, S. Porowski, R.D. Dupuis, and C.J. Eiting, Physica B 273-274, 124 (1999).

5. Z. Liliental-Weber, M. Benamara, J. Washburn, I. Grzegory, S. Porowski, D.J.H. Lambert, C.J. Eiting, and R.D. Dupuis, Appl. Phys. Lett. 75, 4159 (1999).

6. Z. Liliental-Weber, J. Jasinski, M. Benamara, I. Grzegory, S. Porowski, D.J.H. Lambert, C.J. Eiting, and R.D. Dupuis, Phys. Stat. Sol. (b) 228, No. 2, 345 (2001).

7. Z. Liliental-Weber, T. Tomaszewicz, D. Zakharov, J. Jasinski, M.A. O'Keefe, S. Hautakangas, A. Laakso, and K. Saarinen, Mat. Res. Symp. Proc. 798, 711 (2004).

8. P. Vennegues, M. Leroux, S. Dalmasso, M. Benaissa, et al. Phys. Rev. B 68, 235214 (2003).

9. D.P. Bour, H.F. Chung, W. Gotz, L. Romano et al, Mater. Res. Soc. Symp. 449, 509 (1997).

10. A. Thust, W.M.J. Coene, M. Op De Beeck, and D. Van Dyck, Ultramicroscopy 64, 211 (1996). 